\title{
Strong feedback and current noise in nanoelectromechanical systems
}

\author{
O. Usmani, Ya. M. Blanter, and Yu. V. Nazarov \\ Kavli Institute of Nanoscience, Delft University of Technology, Lorentzweg 1, 2628 CJ Delft, The Netherlands
}

(Received 8 March 2006; revised manuscript received 16 February 2007; published 10 May 2007)

\begin{abstract}
We demonstrate the feasibility of a strong feedback regime for a single-electron tunneling device weakly coupled to an underdamped single-mode oscillator. In this regime, mechanical oscillations are generated and the current is strongly modified, whereas the current noise is parametrically large with respect to the Poisson value. This regime requires energy dependence of the tunnel amplitudes. For sufficiently fast tunnel rates, the mechanical contribution to current noise can exceed the Poisson value even beyond the strong feedback regime.
\end{abstract}

DOI: 10.1103/PhysRevB.75.195312

PACS number(s): 73.23.Hk, 72.70.+m

\section{INTRODUCTION}

Recent intensive research on nanoelectromechanical systems (NEMSs) was motivated by a variety of physical effects involved and the prospect of practical applications. ${ }^{1}$ NEMSs have been realized experimentally with molecules, ${ }^{2}$ semiconductor beams, ${ }^{3}$ and suspended carbon nanotubes. ${ }^{4}$ Phenomena observed include negative differential resistance, phonon-assisted transport, and tuning the eigenmodes by the gate voltage. Most of these experiments were performed in the single-electron tunneling (SET) regime. ${ }^{5}$

In this regime, a NEMS is essentially a SET device coupled to a mechanical (harmonic) oscillator. The coupling is provided by a force $F$ (see, e.g., Ref. 6) acting on the oscillator, the value of the force depending on the charge state of the SET device. It determines the dimensionless coupling parameter $\lambda=F^{2} / \hbar M \omega^{3}$, where $M$ and $\omega$ are the mass and the frequency of the oscillator. It was already recognized ${ }^{7}$ that for strong coupling $\lambda \gg 1$, mechanical degrees of freedom strongly influence transport through a SET device, leading, for instance, to polaron physics and FranckCondon effect. However, the weak-coupling regime $\lambda \ll 1$ is characteristic for most of NEMSs and will be considered below.

Naively, the effect of the oscillator on transport current in this regime must be small and proportional to $\lambda$. However, an underdamped oscillator can be swung up to big amplitudes even by a weak random force originated from stochastic electron transfers through the device ${ }^{8}$; this amplitude may provide a strong feedback on the current. A less obvious effect is the extra dissipation due to electron tunneling, ${ }^{9}$ which has been erroneously disregarded in Ref. 8. We demonstrate in this paper that such electron-induced dissipation may become negative, resulting in the generation of mechanical oscillation and in strong mechanical feedback. This takes place if the average charge accumulated in the SET device is a nonmonotonous function of gate voltage.

The strong feedback is the most manifest in the current noise. The natural measure of noise in nanostructures is the Poisson value, ${ }^{10} S_{P}=2 \mathrm{eI}$. We demonstrate that in the strong feedback regime, the noise is always parametrically bigger than $S_{P}$ due to long-time correlations of oscillator amplitude. If the generation is bistable, we predict a telegraph noise that can be exponentially big. Even if the strong feedback is ab- sent, the noise may still exceed $S_{P}$. The experimental observation of the enhanced noise would thus provide a strong evidence for mechanical motion.

\section{SETUP}

SET systems are known to exhibit a (quasi) periodic structure of Coulomb diamonds in the plane of bias $V_{b}$ and gate $V_{g}$ voltages. Inside each diamond, the number of extra electrons $n$ is fixed to an integer. ${ }^{5}$ We concentrate on the region adjacent to the two neighboring diamonds with $n=0$ and $n=1$, where only these two charge states of the SET device participate in transport. This can be accomplished by tuning the gate voltage, if the charging energy is large enough. The system we have in mind is depicted in Fig. 1. It consists of an oscillating island situated between two leads across which a bias voltage $V_{b}$ is applied. It is also coupled to a gate with gate voltage $V_{g}$. Its position and velocity are denoted by $x$ and $v$. The system can be described by the following Hamiltonian:

$$
\hat{H}=\hat{H}_{l}+\hat{H}_{d o t}+\hat{H}_{t}+\hat{H}_{c h},
$$

where

$$
\hat{H}_{l}=\sum_{k} \varepsilon_{k} \hat{c}_{L, k}^{\dagger} \hat{c}_{L, k}+\sum_{k^{\prime}} \varepsilon_{k^{\prime}} \hat{c}_{R, k^{\prime}}^{\dagger} \hat{c}_{R, k^{\prime}}
$$

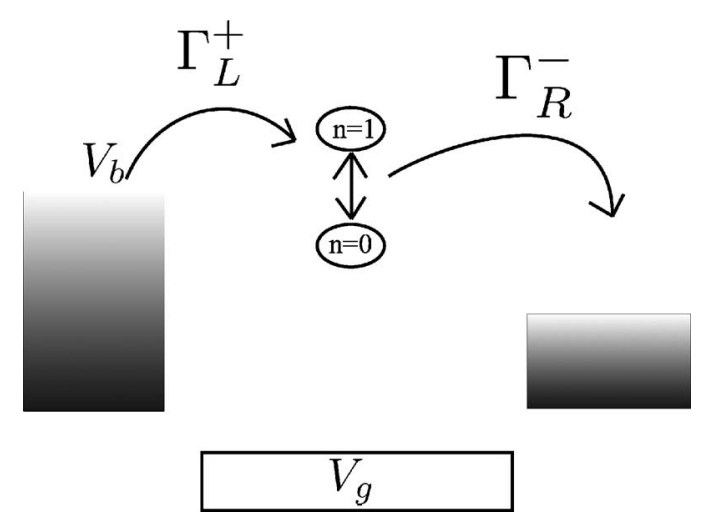

FIG. 1. A schematic view of the system. 


$$
\begin{gathered}
\hat{H}_{d o t}=\hbar \omega\left(\hat{a}^{\dagger} \hat{a}+\frac{1}{2}\right)+F \hat{x} \hat{N}+\sum_{m} \varepsilon_{m} \hat{d}_{m}^{\dagger} \hat{d}_{m}, \\
\hat{H}_{t}=\sum_{k, m} T_{k, m}^{L} \hat{c}_{L, k}^{\dagger} \hat{d}_{m}+\sum_{k^{\prime}, m} T_{k^{\prime}, m}^{R} \hat{c}_{R, k^{\prime}}^{\dagger} \hat{d}_{m}+\text { H.c. }, \\
H_{c h}=\frac{e}{2 C}\left(e \hat{N}+C_{g} V_{g}\right)^{2},
\end{gathered}
$$

$\hat{c}_{(L, R),\left(k, k^{\prime}\right)}^{(\dagger)}$ and $\hat{d}_{m}^{(\dagger)}$ are annihilation (creation) operators of electrons in the left lead, the right lead, and the dot, respectively, and $\varepsilon$ correspond to their energies. The operators $\hat{a}$ and $\hat{a}^{\dagger}$ annihilate and create phonons of the oscillating island. We also have $\hat{N}=\Sigma_{m} \hat{d}_{m}^{\dagger} \hat{d}_{m}$ and $\hat{x}=\sqrt{\hbar / 2 m \omega}\left(\hat{a}^{\dagger}+\hat{a}\right)$. The elements $T$ describe tunneling between the island and the leads, and $C$ is the sum of the capacitances of the left junction and the right junction and the gate capacitance $C_{g}$. The electrons tunnel from the left lead to the island and then from the island to the right lead. We assume that the energy scale of the applied voltages is much larger than the tunneling energy scale. In this case, tunneling is sequential and we can disregard quantum effects such as cotunneling. The energy scale set by the voltages is also assumed to be much larger than the oscillating energy of the island, $\hbar \omega$. In this case, the motion of the island is classical and can be described by its position and velocity. A practical realization of the system can be a molecule or a grain shuttling between electrodes. Another example would consist of a beam (such as a carbon nanotube) singly or doubly clamped between electrodes.

\section{PROBABILITY DISTRIBUTION}

In the classical limit (i.e., the energy scale of the voltages is much larger than the energy scales of the tunneling and oscillating frequencies), the statistical description of the system is provided by the joint distribution function $P_{n}(x, v, t)$. This distribution function obeys the following master equation (see, e.g., Refs. 8 and 11):

$$
\begin{gathered}
\frac{\partial P_{n}}{\partial t}+\left\{v \frac{\partial}{\partial x}+\frac{\partial}{\partial v} \frac{\mathcal{F}}{M}\right\} P_{n}-\operatorname{St}[P]=0, \\
\mathcal{F}=-M \omega^{2} x-\frac{M \omega v}{Q}+F_{n}, \\
\operatorname{St}[P]=(2 n-1)\left[\Gamma^{+}(x) P_{0}-\Gamma^{-}(x) P_{1}\right] .
\end{gathered}
$$

Here, the total force $\mathcal{F}$ acting on the oscillator is the sum of the elastic force, friction force, and charge-dependent coupling force, with respect to the order of terms in Eq. (4). $Q$ $\gg 1$ is the quality factor. We count the position of the oscillator from its equilibrium position in the $n=0$ state. In this case, $F_{n}=n F$.

The "collision integral" $\mathrm{St}[P]$ represents SET. There are four tunnel rates, $\Gamma_{L, R}^{ \pm}$, where the subscripts $L$ and $R$ denote tunneling through the left or right junction, and the superscripts + and - correspond to the tunneling to and from the island, respectively. In Eq. (5), $\Gamma^{ \pm}=\Gamma_{L}^{ \pm}+\Gamma_{R}^{ \pm}$. These rates contain information about the electron levels of the island, which can be continuous (for a metallic island), continuous with an inhomogeneous density, or discrete (for a quantum dot island). It is enough for our purposes to assume that each rate is a function of the corresponding energy cost $\Delta E_{L, R}^{ \pm}$ associated with the addition (removal) of an electron to (from) the island in the state $n=0$ (1) via the left or right junction $\left(\Delta E_{L, R}^{+}=-\Delta E_{L, R}^{-}\right)$. Two independent energy differences are determined by electrostatics, and depend linearly on the voltages. Additionally, they are contributed by the shift of the oscillator,

$$
\Delta E_{L}^{+}=-W+W_{L}-F x, \quad \Delta E_{R}^{-}=-W_{R}+W+F x,
$$

where we introduce a convenient parameter $W$ representing both $e V_{b}$ and $e V_{g}$, with $W_{L}, W_{R}$ lying at the boundaries of the diamonds and $W_{L}<W<W_{R}$ in the transport region. The condition of applicability of the classical approach is that the energy differences are much bigger than energy quantum of the oscillator, $W \gg \hbar \omega$.

To simplify Eq. (3), we implement the separation of the frequency scales: the inverse damping time $\kappa$ of the oscillator, the oscillator frequency $\omega$, and the total tunneling rate $\Gamma_{t}=\Gamma^{+}+\Gamma^{-}$, assuming $\kappa \ll \omega \ll \Gamma_{t}$. The first condition implies that the mechanical energy hardly changes during an oscillation, while the second condition implies that the coordinate varies so slowly that $\Gamma(x)$ hardly changes between two successive tunneling events. In this case, we arrive at a FokkerPlanck equation for the distribution function of the slowest variable-mechanical energy $E-P(E)$. It reads ${ }^{9}$

$$
\frac{\partial P}{\partial t}=\hat{\mathcal{L}} P, \quad \hat{\mathcal{L}} \equiv \frac{\partial}{\partial E}\left[E \kappa(E)+D(E) \frac{\partial}{\partial E}\right] .
$$

Here, $D(E)$ is the diffusion coefficient in energy space and the inverse damping time is given by $\kappa(E)=\widetilde{\kappa}(E)+\omega / Q, \tilde{\kappa}$ being the SET contribution. It is instructive to express those parameters in terms of the average number of extra electrons in the island, $\bar{n}(x) \equiv \Gamma^{+} / \Gamma_{t}$,

$$
\left\{\begin{array}{c}
D(E) / E \\
\widetilde{\kappa}(E)
\end{array}\right\}=\frac{F^{2}}{M}\left\langle\frac{1}{\Gamma_{t}}\left\{\begin{array}{c}
\bar{n}(1-\bar{n}) \\
\partial \bar{n} / \partial W
\end{array}\right\}\right\rangle .
$$

Here, the angular brackets denote an average over the oscillation period, $\langle A(x)\rangle=\int(d \theta / \pi) \cos ^{2} \theta A(x(E) \sin \theta)$, the oscillation amplitude being given by $x(E)=\sqrt{2 E / M} / \omega$.

The SET contribution to the damping $\widetilde{\kappa}$ has been erroneously disregarded in Ref. 8. In fact, as Eq. (7) suggests, the diffusion and damping are closely related. In particular, in the absence of bias $\left(W_{L}=W_{R}\right)$ the average number of electrons is determined by the Boltzmann distribution and one proves that $d \bar{n} / d W=\bar{n}(1-\bar{n}) / k_{B} T$. In this case, the diffusion coefficient obeys the Einstein relation $D(E)=k_{B} T E \kappa(E)$. This, in its own turn, guarantees that Eq. (6) is satisfied with the Boltzmann distribution $P(E) \propto \exp \left(-E / k_{B} T\right)$. At $e V_{b}$ $\gg k_{B} T$, the Einstein relation does not hold anymore. The effective temperature $E D / \kappa$ of the oscillator may become of the order of $e V_{b}$. Moreover, we will demonstrate that for energy-dependent tunneling rates, the damping $\widetilde{\kappa}$ can be- 
come negative. This signals instability with respect to interaction with the oscillator.

The stationary solution of Eq. (6) apart from a normalization constant reads

$$
P(E) \propto \exp \left[-\int_{0}^{E} d E^{\prime} E^{\prime} \kappa\left(E^{\prime}\right) / D\left(E^{\prime}\right)\right] .
$$

We see that the contribution $\omega / Q$ in $\kappa$ is only important provided $Q^{-1} \ll(\hbar \omega \lambda / W)\left(\omega / \Gamma_{t}\right)$. To stress the importance of the SET contribution, we will disregard other contributions to the damping $(Q \rightarrow \infty)$, so that $\widetilde{\kappa}=\kappa$.

The current is modified by mechanical motion. At a given mechanical energy $E$, the current averaged over the oscillation period, $I_{W}(E)$, is determined from the dependence of the current on the energy parameter, $I(W)$ $\equiv \Gamma_{L}^{+}(W) \Gamma_{R}^{-}(W) / \Gamma_{t}(W)$, in the absence of oscillations: $I_{W}(E)$ $\equiv \int(d \theta / 2 \pi) I(W+F x(E) \sin \theta)$. In the limit of small amplitudes, one has $I_{W}(E) \approx I(W)+I^{\prime \prime}(W) \lambda \hbar \omega E / 2$. The actual current $I_{W}$ is obtained by averaging $I_{W}(E)$ over $E$ with the distribution function $P(E)$. Zero-frequency current noise in the Fokker-Planck framework is obtained as

$$
S=-4 \int_{0}^{\infty} d E \delta I_{W}(E) \hat{\mathcal{L}}^{-1} \delta I_{W}(E) P(E),
$$

with $\delta I_{W}(E) \equiv I_{W}(E)-I_{W}$. In our assumptions, the distribution function is sharp at the energy scale of interest. Indeed, the typical mechanical energy needed to modify the rates is determined from the relation $e V_{b} \simeq F x(E)$, yielding $E$ $\simeq M \omega^{2}\left(e V_{b} / F\right)^{2}$. If $\lambda \ll 1$, this always exceeds the typical energy fluctuation $e V_{b}$. If the damping is positive at all $E, P$ has a sharp maximum at $E=0$ and the current is very close to $I_{W}(0)$. The average amplitude of the oscillations is too small to induce a noticeable mechanical feedback.

The situation changes drastically if $\kappa(E)$ becomes negative, indicating instability and growing amplitude of the oscillations. Since $\kappa(E)$ is determined by the tunnel rates only, the amplitude growth can only be stabilized by significant modification of the rates by the amplitude growing: This is the strong mechanical feedback. Positions of probability maxima are determined by the roots of

$$
E \kappa(E)=0 .
$$

A nontrivial root $E_{0} \neq 0$ indicates a generation of mechanical oscillation with almost constant amplitude. This may strongly modify the current that is now given by $I_{W}\left(E_{0}\right)$. Our analysis shows that the negative damping can only arise from the energy dependence of the tunnel amplitudes. This dependence is intrinsic for both semiconductor quantum dots and molecules.

\section{ILLUSTRATIVE EXAMPLE}

\section{A. Characteristics of the system}

To illustrate, we have chosen exponential energy dependence typical for wide tunnel barriers ${ }^{12,14}$ and one electron level in the SET system,

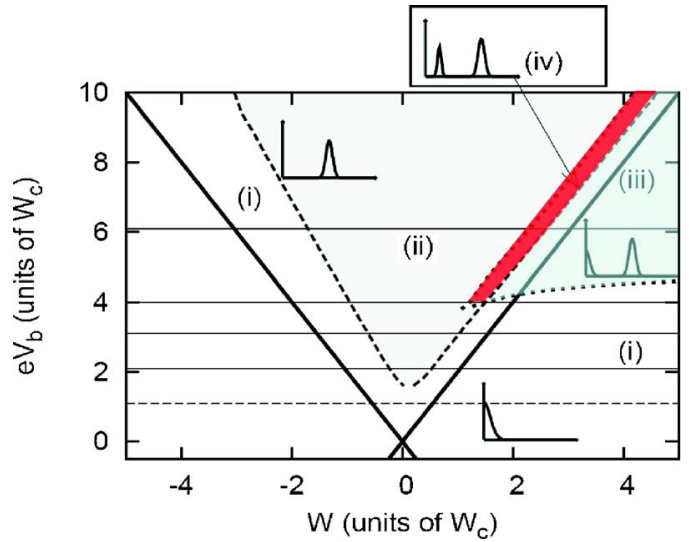

FIG. 2. (Color online) Four stability regions in the gate-bias voltage plane. The bold solid lines indicate the edge of the Coulomb diamonds. The insets show the sketch of $P(E)$ in each region. The horizontal lines indicate bias voltages used for current and noise scans in Figs. 3 and 4.

$$
\begin{gathered}
\Gamma_{L, R}^{+}=2 \Gamma_{L, R}^{0} e^{-a_{L, R} \Delta E_{L, R}^{+}\left[1-f_{F}\left(-\Delta E_{L, R}^{+}\right)\right],} \\
\Gamma_{L, R}^{-}=\Gamma_{L, R}^{0} e^{a_{L, R} \Delta E_{L, R}^{-}} f_{F}\left(\Delta E_{L, R}^{-}\right),
\end{gathered}
$$

the factor 2 accounting for the spin degeneracy of the state $n=1$. The energy dependence sets a new energy scale $W_{c}$, smaller than the charging energy. For concrete illustration, we choose $a_{L}=0.3, a_{R}=0.75, k_{B} T=0.2 W_{c}$, and $\Gamma_{L}^{0}=\Gamma_{R}^{0}$. To give a value for the energy scale $W_{c}$, we take a system with $\Gamma^{0}=125 \mathrm{GHz}$ and $\omega=10 \mathrm{GHz}$, with barrier heights of 11 and $26 \mathrm{meV}$ for the left and right barriers with respective widths of 2.2 and $2.8 \mathrm{~nm}$. In this case, we get $W_{c} \approx 5 \mathrm{meV}$ and $T$ $\approx 8 \mathrm{~K}$. The quality factor $Q=10^{5}$ is sufficient to observe the effects.

\section{B. Probability distribution}

Figure 2 presents the regions in gate-bias voltage plane that differ by number and stability of the roots of Eq. (10). Region (i) corresponds to positive damping at all $E$ and the absence of strong mechanical feedback. In region (ii), the only stable solution corresponds to the generation of mechanical oscillations. There is bistability in regions (iii) (stable roots at $E=0$ and a finite amplitude) and (iv) (two stable roots at finite amplitudes). Strong mechanical feedback is present in regions (ii), (iii), and (iv). It is remarkable that region (iii) eventually extends to the Coulomb diamond where no current is possible at zero temperature without the oscillator: Generation of mechanical oscillation makes it possible.

\section{Current}

We illustrate the modification of the current by mechanical motion in Fig. 3. The modification is noticeable provided the generation of oscillations takes place. It can be of the same order of magnitude as the unmodified current and of either sign. The current can even exhibit jumps if there are at least two stable values, $E_{1}$ and $E_{2}$, of the amplitude gener- 
(a) $e V_{b} / W_{c}=2.1$

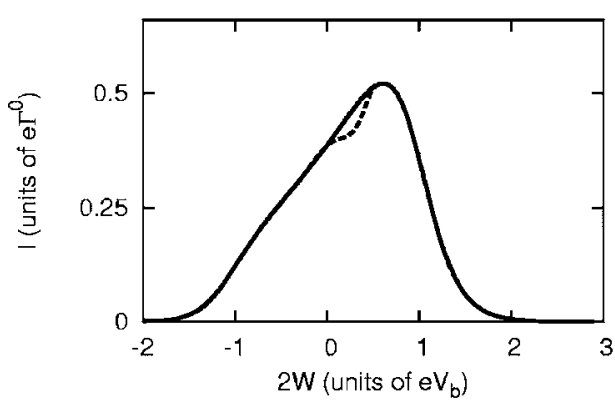

(c) $e V_{b} / W_{c}=4$

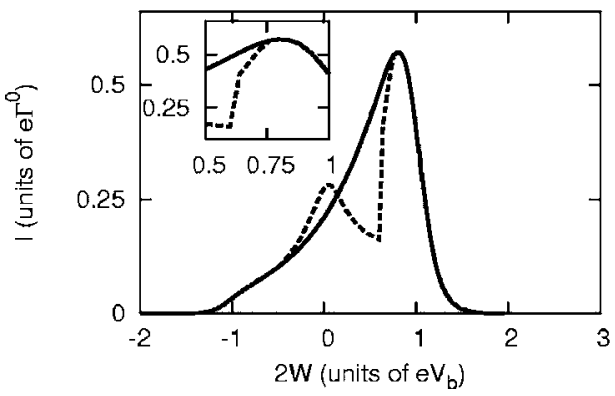

(b) $e V_{b} W_{c}=3.1$

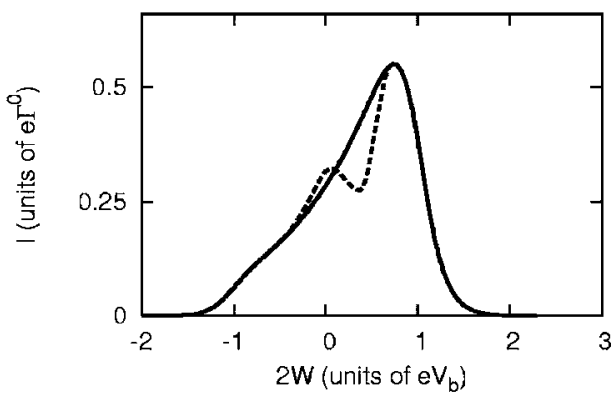

(d) $e V_{b} W_{c}=6.1$

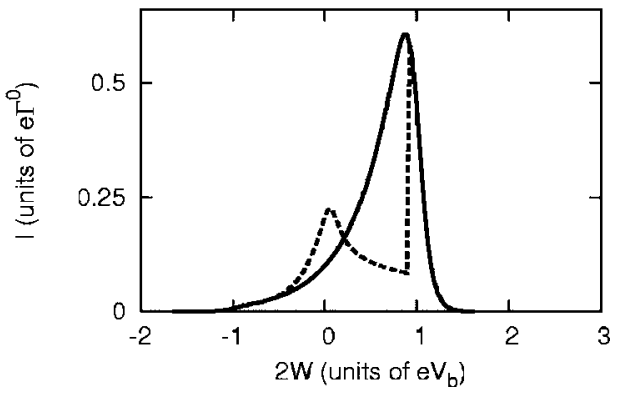

FIG. 3. Current modification in strong feedback regime for different bias voltages. The dashed (solid) lines give the current modified (unmodified) by mechanical motion. (a) and (b) demonstrate that the modification is restricted to region (ii) where the generation takes place. (c) and (d) illustrate the bistable regions (iii) and (iv). In (c), the current in region (iv) gives a jump where the probabilities of two stable amplitudes are the same. To the right of the jump, the lower amplitude value is more probable. This value decreases and becomes zero at the border of region (iii). Therefore, the modification of the current ceases there (see inset). In (d), the probabilities become the same in region (iii). Therefore, the modification ceases immediately after the jump.

ated. The position of the jump corresponds to the values of $W$ at which the probabilities $P\left(E_{1}\right)$ and $P\left(E_{2}\right)$ are equal.

\section{Noise}

Let us now turn to the current noise. First, we evaluate the noise in the absence of feedback [region (i) in Fig. 2]. We make use of Eq. (9) approximating $D(E), \kappa(E)$, and $\delta I_{W}(E)$ by their values at $E \rightarrow 0$. This yields

$$
S=\left.\frac{F^{4}}{M^{2} \omega^{4}}\left(\frac{\partial^{2} I}{\partial W^{2}}\right)^{2} \frac{D^{2}(E)}{\kappa^{3}(E) E^{2}}\right|_{E=0} .
$$

The ratio of the electromechanical noise and the Poisson value $S_{P}$ is of the order of

$$
\frac{S}{S_{P}} \sim\left(\frac{\Gamma^{0}}{\omega}\right)^{2} \frac{\hbar \omega \lambda}{W} .
$$

The small value of the second factor can be compensated by the large value of the first one. In this case, the electromechanical noise, concentrated at frequencies of the order of $\kappa$, exceeds the Poisson value.

In region (ii), where the stable generation of the oscillation with the energy $E_{0}$ takes place, the current noise is due to small fluctuations of the oscillation amplitude. These fluctuations occur at a frequency scale of the order of $\kappa^{\prime}\left(E_{0}\right) E_{0}$. The noise is given by

$$
S=4\left(\frac{I^{\prime 2}\left(E_{0}\right) D\left(E_{0}\right)}{E_{0}^{2} \kappa^{\prime 2}\left(E_{0}\right)}\right), \quad \frac{S}{S_{P}} \sim\left(\frac{\Gamma^{0}}{\omega}\right)^{2} .
$$

That is, it exceeds the Poisson value by a large factor. Our numerical results for regions (i) and (ii) [Figs. 4(a) and 4(b)] show that Eqs. (13) and (14) give a scale of the noise rather than a good estimation. The actual values of noise change by 3-4 orders of magnitude. The reason for that is that the parameters $I^{\prime}, I^{\prime \prime}, \kappa$, and $\kappa^{\prime}$ may become close to zero.

In regions (iii) and (iv), the oscillation amplitude randomly switches between two values $E_{1}$ and $E_{2}$. Since they correspond to two distinct values of the current $I_{1}$ and $I_{2}$, a telegraph noise is observed. The distribution function reaches maxima at $E_{1,2}$, while the switching corresponds to passing the minimum of the distribution function $P\left(E_{m}\right)$. The switching times are therefore exponentially long. This may lead to exponentially large enhancement of noise. We compute the switching rates by Kramers' method, ${ }^{13}$ 
(a) $e V_{b} / W_{c}=1.1$

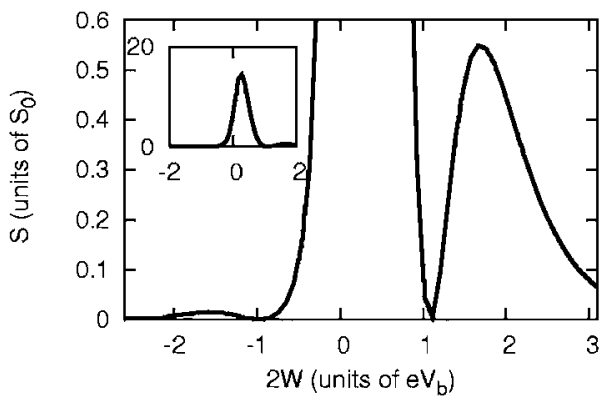

(c) $\mathrm{eV}_{\mathrm{b}} / \mathrm{W}_{\mathrm{c}}=4$

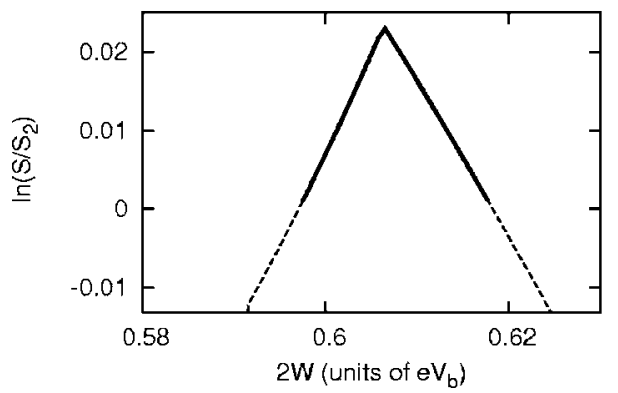

(b) $\mathrm{eV}_{\mathrm{b}} \mathrm{W}_{\mathrm{c}}=2.2$

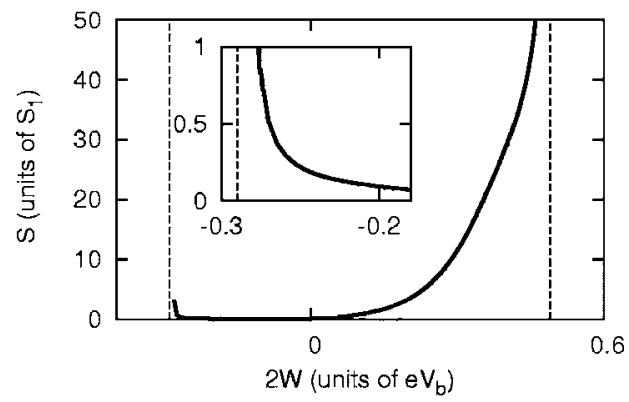

(d) $e V_{b} / W_{c}=6.1$

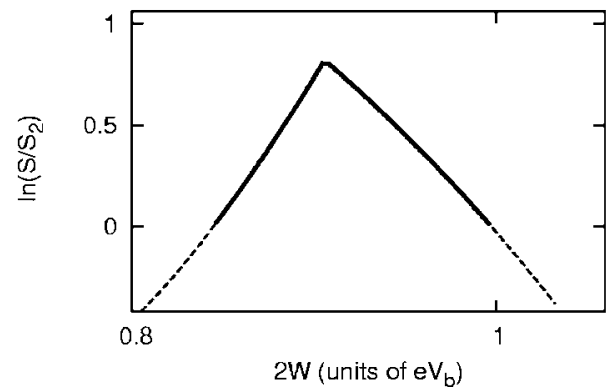

FIG. 4. Mechanical contribution to current noise for different bias voltages. (a) Noise in stable region (i) [Eq. (12)] becomes zero at $I^{\prime \prime}=0$. (b) Noise in region (ii) changes by orders of magnitude approaching zero at $I^{\prime}=0$ and diverging at the boundaries of the region where $E_{0} \rightarrow 0$ [Eq. (14)]. [(c) and (d)] Telegraph noise is presented in the bistable regions only. The solid lines indicate the region where it is exponentially large. The cusp at maximum corresponds to equal probabilities of two stable amplitude values (as discussed, the current gives a jump at this point). The noise scales are $S_{0}=\lambda \hbar\left(\Gamma^{0}\right)^{3} / \omega W_{c}, S_{1}=\left(\Gamma^{0}\right)^{3} / \omega^{2}$, and $S_{2}=\left(\Gamma^{0}\right)^{3} W_{c} / \lambda \hbar \omega^{3} \exp \left(W_{c} / \lambda \hbar \omega\right)$.

$$
\begin{gathered}
\Gamma^{1 \rightarrow 2,2 \rightarrow 1}=\frac{E_{m} \kappa^{\prime}\left(E_{m}\right)}{2 \pi} \sqrt{\frac{\gamma\left(E_{1,2}\right)}{\gamma\left(E_{m}\right)}} \frac{P\left(E_{m}\right)}{P\left(E_{1,2}\right)}, \\
\gamma(E) \equiv E \kappa^{\prime}(E) / D(E), \quad E \neq 0 \\
\gamma(0)=2 \pi E^{2} \kappa^{2}(E) /\left.D^{2}(E)\right|_{E=0} .
\end{gathered}
$$

These rates enter the following master equation for the probabilities to be in states 1 and $2, P_{1}$ and $P_{2}$ :

$$
\frac{\partial P_{1}}{\partial t}=-\Gamma^{1 \rightarrow 2} P_{1}+\Gamma^{2 \rightarrow 1} P_{2}
$$

It has to be combined with the condition that the sum of both probabilities is 1 . The stationary solutions are given by

$$
P_{1}=\frac{\Gamma^{2 \rightarrow 1}}{\Gamma^{1 \rightarrow 2}+\Gamma^{2 \rightarrow 1}}, \quad P_{2}=\frac{\Gamma^{1 \rightarrow 2}}{\Gamma^{1 \rightarrow 2}+\Gamma^{2 \rightarrow 1}} .
$$

The average stationary current yields

$$
\langle I\rangle=I_{1} P_{1}+I_{2} P_{2}=\frac{I_{1} \Gamma^{2 \rightarrow 1}+I_{2} \Gamma^{1 \rightarrow 2}}{\Gamma^{1 \rightarrow 2}+\Gamma^{2 \rightarrow 1}} .
$$

Another quantity of interest is the average of the square of the current fluctuations,

$$
\begin{aligned}
\left\langle(\delta I)^{2}\right\rangle & =\left\langle(I-\langle I\rangle)^{2}\right\rangle \\
& =P_{1} P_{2}\left(I_{1}-I_{2}\right)^{2} \\
& =\frac{\Gamma^{1 \rightarrow 2} \Gamma^{2 \rightarrow 1}}{\left(\Gamma^{1 \rightarrow 2}+\Gamma^{2 \rightarrow 1}\right)^{2}}\left(I_{1}-I_{2}\right)^{2} .
\end{aligned}
$$

In order to obtain the noise, one needs to look at the correlator $\langle\delta I(t) \delta I(0)\rangle=\Sigma_{l, m} \delta I_{l} \delta I_{m} P_{l m}(t) P_{m}$, which corresponds to all possibilities of having state $m$ at time zero and subsequently state $l$ at time $t$. To obtain it, we need to compute the probability $P_{l m}(t)$ to be in state $l$ at time $t$, given that the system was in state $m$ at time 0 . It is a (time-dependent) solution of the master equation, with $P_{l m}=\delta_{l m}$ as an initial condition. It is given by

$$
P_{l m}(t)=\left(\delta_{l m}-P_{l}\right) e^{-\left(\Gamma^{1 \rightarrow 2}+\Gamma^{2 \rightarrow 1}\right)|t|}+P_{l},
$$

where $P_{l}$ is the stationary solution to be in state $l$, and where the absolute value on the time comes for time-reversal symmetry reasons. Calculating the sums in the correlator and using $\Sigma_{l} \delta I_{l} P_{l}=\langle\delta I\rangle=0$, we get

$$
\langle\delta I(t) \delta I(0)\rangle=e^{-\left(\Gamma^{1 \rightarrow 2}+\Gamma^{2 \rightarrow 1}\right)|t|}\left\langle(\delta I)^{2}\right\rangle .
$$

Inserting this in the following definition for the current noise, $S(\omega)=2 \int_{-\infty}^{\infty} e^{-i \omega t}\langle\delta I(t) \delta I(0)\rangle d t$ (the factor 2 is there to symmetrize the noise), we obtain the telegraph noise

$$
S(\omega)=4\left(I_{1}-I_{2}\right)^{2} \frac{\Gamma^{1 \rightarrow 2} \Gamma^{2 \rightarrow 1}}{\left(\Gamma^{1 \rightarrow 2}+\Gamma^{2 \rightarrow 1}\right)\left[\omega^{2}+\left(\Gamma^{1 \rightarrow 2}+\Gamma^{2 \rightarrow 1}\right)^{2}\right]} .
$$

Looking at the zero-frequency limit, we get 


$$
S(0)=4\left(I_{1}-I_{2}\right)^{2} \frac{\Gamma^{1 \rightarrow 2} \Gamma^{2 \rightarrow 1}}{\left(\Gamma^{1 \rightarrow 2}+\Gamma^{2 \rightarrow 1}\right)^{3}},
$$

that is exponentially large provided $P\left(E_{1}\right) P\left(E_{2}\right) \gtrsim P^{2}\left(E_{m}\right)$ [Figs. 4(c) and 4(d)]. While this condition is not satisfied at the edges of bistability region, it certainly holds near the current jump, $P\left(E_{1}\right) \approx P\left(E_{2}\right)$, where the noise reaches maximum. The estimation reads

$$
\ln \left[\frac{S}{S_{P}} \frac{\hbar \omega \lambda}{W}\left(\frac{\omega}{\Gamma^{0}}\right)^{2}\right] \sim \frac{W}{\hbar \omega \lambda} .
$$

\section{CONCLUSIONS}

To conclude, we analyzed the SET system weakly coupled to a mechanical oscillator and proved the existence of significant modification of the current under condition of strong feedback where generation of mechanical oscillation takes place. The latter is feasible for energy-dependent tunneling amplitudes. The current noise generated by mechanical motion in the strong feedback regime significantly exceeds the Poisson value and may be exponentially large if the generation is bistable. Even if no generation takes place, this extra noise may exceed $S_{P}$ for sufficiently fast tunneling rates.

\section{ACKNOWLEDGMENTS}

This work was supported by the Netherlands Foundation for Fundamental Research on Matter (FOM) and EC FP6 funding (Contract No. FP6-2004-IST-003673).
${ }^{1}$ A. N. Cleland, Foundations of Nanomechanics (Springer, Heidelberg, 2002).

${ }^{2}$ H. Park, J. Park, A. K. L. Lim, E. H. Anderson, A. P. Alivisatos, and P. L. McEuen, Nature (London) 407, 57 (2000); E. S. Soldatov, V. V. Khanin, A. S. Trifonov, D. E. Presnov, S. A. Yakovenko, G. B. Khomutov, C. P. Gubin, and V. V. Kolesov, JETP Lett. 64, 556 (1996); S. Kubatkin, A. Danilov, M. Hjort, J. Cornil, J.-L. Brédas, N. Stuhr-Hansen, P. Hedegård, and T. Bjørnholm, Nature (London) 425, 698 (2003); R. H. M. Smit, Y. Noat, C. Untiedt, N. D. Lang, M. C. van Hemert, and J. M. van Ruitenbeek, ibid. 419, 906 (2002).

${ }^{3}$ R. G. Knobel and A. N. Cleland, Nature (London) 424, 291 (2003); E. M. Weig, R. H. Blick, T. Brandes, J. Kirschbaum, W. Wegscheider, M. Bichler, and J. P. Kotthaus, Phys. Rev. Lett. 92, 046804 (2004); M. D. LaHaye, O. Buu, B. Camarota, and K. C. Schwab, Science 304, 74 (2004).

${ }^{4}$ S. Sapmaz, P. Jarillo-Herrero, Ya. M. Blanter, C. Dekker, and H. S. J. van der Zant, Phys. Rev. Lett. 96, 026801 (2006); B. J. LeRoy, S. G. Lemay, J. Kong, and C. Dekker, Nature (London) 432, 371 (2004); V. Sazonova, Y. Yaish, H. Üstünel, D. Roundy, T. A. Arias, and P. L. McEuen, ibid. 431, 284 (2004).

${ }^{5}$ M. H. Devoret and H. Grabert, in Single Charge Tunneling, edited by H. Grabert and M. H. Devoret, NATO Advanced Studies
Institute, Series B: Physics (Plenum, New York, 1992), Vol. 294, p. 1; G.-L. Ingold and Yu. V. Nazarov, ibid. p. 21.

${ }^{6}$ S. Sapmaz, Ya. M. Blanter, L. Gurevich, and H. S. J. van der Zant, Phys. Rev. B 67, 235414 (2003).

${ }^{7}$ S. Braig and K. Flensberg, Phys. Rev. B 68, 205324 (2003); A. Mitra, I. Aleiner, and A. J. Millis, ibid. 69, 245302 (2003); J. Koch and F. von Oppen, Phys. Rev. Lett. 94, 206804 (2005); D. Mozyrsky, M. B. Hastings, and I. Martin, Phys. Rev. B 73, 035104 (2006).

${ }^{8}$ Ya. M. Blanter, O. Usmani, and Yu. V. Nazarov, Phys. Rev. Lett. 93, 136802 (2004).

${ }^{9}$ Ya. M. Blanter, O. Usmani, and Yu. V. Nazarov, Phys. Rev. Lett. 94, 049904(E) (2005).

${ }^{10}$ Ya. M. Blanter and M. Büttiker, Phys. Rep. 336, 1 (2000).

${ }^{11}$ A. D. Armour, Phys. Rev. B 70, 165315 (2004); A. Isacsson and T. Nord, Europhys. Lett. 66, 708 (2004); A. A. Clerk and S. Bennett, New J. Phys. 7, 238 (2005).

${ }^{12}$ A. N. Korotkov and Y. V. Nazarov, Physica B 173, 217 (1991).

${ }^{13}$ H. A. Kramers, Physica (Amsterdam) 7, 284 (1940); W. Fuller Brown, Jr., Phys. Rev. 130, 1677 (1963); C. W. Gardiner, Handbook of Stochastic Methods (Springer, Berlin, 2002).

${ }^{14}$ A. Bezryadin, C. Dekker, and G. Schmid, Appl. Phys. Lett. 71, 1273 (1997). 